International Journal of Applied Mathematics

Volume 34 No. $6 \quad 2021,1123-1140$

ISSN: $1311-1728$ (printed version); ISSN: 1314-8060 (on-line version)

doi: http://dx.doi.org/10.12732/ijam.v34i6.6

\title{
ESTIMATION OF THE INTENSITY OF THE SUCCESSIVE INSTANTANEOUS EMISSIONS RELEASED BY AN AIR POLLUTION POINT SOURCE
}

\author{
D. Parra-Guevara ${ }^{1}$, Yu. N. Skiba ${ }^{2}$ \\ Institute of Atmospheric Sciences and Climate Change \\ National Autonomous University of Mexico \\ Circuito Exterior, Ciudad Universitaria \\ CDMX, C. P. 04510, MEXICO
}

\begin{abstract}
A new method for assessing the intensity of successive instantaneous emissions of an air pollutant from a point source is presented. The intensity parameters were obtained using a time series of the pollutant concentration detected at a monitoring site. A well-posed atmospheric dispersion model is used to estimate the transport of the pollutant from the source. The parameter estimation method is formulated as an optimization problem. The optimal point determines the intensity of instantaneous emissions and is calculated as the solution of a positive system of linear equations. Particular solutions of the dispersion model are used to adjust the matrix and the rightside of the linear system. The capabilities of the parameter estimation method are demonstrated using one-dimensional synthetic numerical examples.
\end{abstract}

AMS Subject Classification: 35K20, 90C20, $15 \mathrm{~A} 06$

Key Words: dispersion model; parameter estimation; optimization; positive linear systems

\section{Introduction}

The emission rate of a pollution source is fundamental information for the environmental research. In particular, the air quality models use such information

Received: May 5, 2021

(C) 2021 Academic Publications

${ }^{\S}$ Correspondence author 
to forecast the pollution levels in a region at different time scales. This makes it possible to carefully assess the risk to the population and take the necessary measures in advance to combat air pollution $[13,15]$. As a consequence, the development of methods for assessing the intensity of emissions of pollutants is of paramount importance for emergency assessment and response to the impact of hazardous substances accidentally released into the atmosphere as a result of accidents at industrial enterprises and nuclear power plants $[1,9]$. It is important to note that the estimation of emission rates is often an ill-posed problem for which there is no general method for solving, and each developed method solves the estimation problem taking into account certain simplifications in the system [2].

This work presents a new method for assessing the emission rate $Q(t)$ of a point source of air pollution. It is assumed that the source emits a pollutant at a known point $\mathbf{r}_{0}$, at a rate $Q(t)$ in the form of $N$ successive instantaneous emissions:

$$
Q(t)=\sum_{i=1}^{N} Q_{i} \delta\left(t-t_{i}\right),
$$

where the Dirac function $\delta$ sets a unit impulse at each known instant $t_{i}$. The parameter estimation problem consists in the approximation of the intensity coefficients $Q_{i}$. This problem is solved using the time series of the pollutant concentration observed at the monitoring site. The method is formulated as minimization of a quadratic function, and the emission rates $Q_{i}$ are obtained as the solution of a positive system of linear equations of size $N \times N$. The transport of the pollutant in the atmosphere is estimated by means of a wellposed linear dispersion model. Particular solutions of the dispersion model are used to calculate the matrix and the right-hand side of the positive system. The three-dimensional dispersion model is described in Section 2. Some useful properties related to the solution of positive systems of linear equations and its sensitivity are presented in Section 3. The formulation of the parameter estimation method is presented in Section 4. Finally, Section 5 contains several synthetic numerical examples using a one-dimensional version of the dispersion model.

\section{Dispersion model}

In this section, a three-dimensional linear dispersion model is briefly described [10]. The model governs the dispersion of a primary pollutant emitted into the atmosphere by point, line or area sources. It is useful for establishing a 
linear relationship between emissions and concentrations of a single (passive) pollutant such as $\mathrm{CO}, \mathrm{SO}_{2}, \mathrm{NO}_{x}$ or soot [3].

Let $\mathbf{D}=D \times(0, H)$ be a simply connected bounded domain in $\mathbf{R}^{3}$ with the boundary $\partial \mathrm{D}=S_{0} \cup S \cup S_{H}$ which is the union of the cylindric lateral surface $S$, the base of the domain $S_{0}$ (generally not horizontal), and top cover $S_{H}$ at $z=H$. The dispersion model for forecasting the concentration $\phi(\mathbf{r}, t)$ of a primary pollutant in the domain $\mathbf{D}$ and short-time interval $(0, T)$ is defined by the following equations:

$$
\begin{gathered}
\frac{\partial \phi}{\partial t}+\mathbf{U} \cdot \nabla \phi+\sigma \phi-\nabla \cdot(\mu \nabla \phi)-\frac{\partial}{\partial z} \mu_{z} \frac{\partial \phi}{\partial z}+\nabla \cdot \phi^{s}=f(\mathbf{r}, t), \\
\phi^{s}=-\nu^{s} \phi \mathbf{e}_{3} \quad \text { in } \quad \mathbf{D}, \\
\phi(\mathbf{r}, 0)=\phi^{0}(\mathbf{r}) \text { in } \mathbf{D}, \\
\mu \nabla \phi \cdot \mathbf{n}-U_{n} \phi=0 \quad \text { on } \quad S^{-}, \\
\mu \nabla \phi \cdot \mathbf{n}=0 \text { on } S^{+}, \\
\widehat{\mu} \nabla \phi \cdot \mathbf{n}=0 \text { on } S_{0}, \\
\mu_{z} \frac{\partial \phi}{\partial z}-U_{n} \phi=-\nu^{s} \phi \quad \text { on } \quad S_{H}^{-}, \\
\mu_{z} \frac{\partial \phi}{\partial z}=-\nu^{s} \phi \quad \text { on } \quad S_{H}^{+}, \\
\nabla \cdot \mathbf{U}=\frac{\partial u}{\partial x}+\frac{\partial v}{\partial y}+\frac{\partial w}{\partial z}=0 \quad \text { in } \quad \mathbf{D} .
\end{gathered}
$$

Here $\phi^{0}(\mathbf{r}) \geq 0$ is the distribution of the pollutant in the domain $\mathbf{D}$ at the initial moment $t=0, \sigma(\mathbf{r}, t) \geq 0$ is the chemical transformation coefficient, and $\mu(\mathbf{r}, t)>0$ and $\widehat{\mu}(\mathbf{r}, t)>0$ are the turbulent diffusion arrays:

$$
\begin{gathered}
\mu=\left(\begin{array}{ll}
\mu_{x}(\mathbf{r}, t) & 0 \\
0 & \mu_{y}(\mathbf{r}, t)
\end{array}\right) \quad \text { and } \\
\widehat{\mu}=\left(\begin{array}{lll}
\mu_{x}(\mathbf{r}, t) & 0 & 0 \\
0 & \mu_{y}(\mathbf{r}, t) & 0 \\
0 & 0 & \mu_{z}(\mathbf{r}, t)
\end{array}\right) .
\end{gathered}
$$

In equation (1), the term $\nabla \cdot \phi^{s}$ describes the change in pollutant concentration because of sedimentation at a constant rate $\nu^{s}>0$. It is assumed that the wind velocity $\mathbf{U}(\mathbf{r}, t)=(u, v, w)$ is known in $\mathbf{D}$ and satisfies the continuity equation (9). 
Assume that the forcing

$$
f(\mathbf{r}, t)=\sum_{i=1}^{M} f_{i}(\mathbf{r}, t),
$$

is formed by point, line or area emission sources $f_{i}(\mathbf{r}, t)$ located in the domain $\mathbf{D}$, $i=1, \ldots, M$. In particular, each point emission source $f_{i}(\mathbf{r}, t)$ can be described through its emission rate $Q_{i}(t)$ and emission site $\mathbf{r}_{i}$, that is, $f_{i}(\mathbf{r}, t)=Q_{i}(t) \delta(\mathbf{r}-$ $\left.\mathbf{r}_{i}\right)$, where $\delta\left(\mathbf{r}-\mathbf{r}_{i}\right)$ is the Dirac delta centered at $\mathbf{r}_{i} \in \mathbf{D}$. On the other hand, the domain of function $f_{i}(\mathbf{r}, t)$ is reduced to a line $\Gamma_{i} \subset D$ in the case of a linearly distributed source, and to a $2 \mathrm{D}$ region $A_{i} \subset D$ in the case of a surface source $f_{i}(\mathbf{r}, t)$. It is important to note that each linearly distributed source, as well as each source distributed over an area, can be approximated by the sum of point sources [11].

The conditions set on the open boundary $\partial \mathbf{D}$ of domain $\mathbf{D}$ lead to the wellposed problem in the sense of Hadamard [4]. The boundary $S$ is divided into the outflow part $S^{+}$where $U_{n} \geq 0$ (advective pollution flow is directed out of D) and the inflow part $S^{-}$where $U_{n}<0$ (advective pollution flow is directed into $\mathbf{D}$ ). Here $U_{n}=\mathbf{U} \cdot \mathbf{n}$ denotes the projection of the velocity vector $\mathbf{U}$ on the outward unit normal $\mathbf{n}$ to $S$. The region $\mathbf{D}$ is assumed to be large enough to include all important pollution sources. Thus, it is supposed that there is no sources outside $\mathbf{D}$, and by condition (4), the total (diffusion plus advective) flow of pollutant is zero through the inflow part $S^{-}$. The flow of pollutant is nonzero only through the outflow part $S^{+}$, besides, according to (5), the diffusion flow of pollutant through $S^{+}$is assumed to be negligible as compared with the corresponding advective flow. Equation (6) indicates no flow of pollutant through the base $S_{0}$, since $\mathbf{U} \cdot \mathbf{n}$ and $\nu^{s}$ are both zero at $S_{0}$. The conditions (7) and (8) have a similar meaning at the boundary $S_{H}$, where the sedimentation of particles is additionally taken into account. In general, equations (7) and (8) are necessary because $w=0$ on $S_{0}$ and therefore condition (9) leads to a non-zero vertical velocity component at $S_{H}$ :

$$
w(x, y, z, t)=-\int_{0}^{z}\left(\frac{\partial u}{\partial x}+\frac{\partial v}{\partial y}\right) d z .
$$

With such boundary conditions, problem (1) - (9) is mathematically wellposed in the sense of Hadamard [4], i.e., its solution exists, is unique and continuously depends on the initial condition and forcing [14]. This follows from the fact that the problem operator

$$
A \phi=\mathbf{U} \cdot \nabla \phi+\sigma \phi-\nabla \cdot(\mu \nabla \phi)-\frac{\partial}{\partial z} \mu_{z} \frac{\partial \phi}{\partial z}+\nabla \cdot \phi^{s},
$$


is nonnegative:

$$
\begin{aligned}
& (A \phi, \phi)=\int_{\mathbf{D}}\left\|\widehat{\mu}^{\frac{1}{2}} \nabla \phi\right\|_{2}^{2} d \mathbf{r}+\int_{\mathbf{D}} \sigma \phi^{2} d \mathbf{r}+\frac{1}{2} \int_{S_{0}} \nu^{s} \phi^{2}\left|\mathbf{e}_{3} \cdot \mathbf{n}\right| d S \\
& +\int_{S_{H}} \nu^{s} \phi^{2} d S+\frac{1}{2}\left\{\int_{S^{+} \cup S_{H}^{+}} U_{n} \phi^{2} d S-\int_{S^{-} \cup S_{H}^{-}} U_{n} \phi^{2} d S\right\} \geq 0 .
\end{aligned}
$$

Here $(\phi, \eta)=\int_{\mathbf{D}} \phi \eta d \mathbf{r}$ and $\|\phi\|_{2}=\left(\int_{\mathbf{D}} \phi^{2} d \mathbf{r}\right)^{1 / 2}$ define the inner product and the norm in the Hilbert space $L_{2}(\mathbf{D})$, respectively. Therefore, it can be shown [14] that

$$
\|\phi\|_{2} \leq T \max _{0 \leq t \leq T}\|f(\mathbf{r}, t)\|_{2}+\left\|\phi^{0}\right\|_{2} .
$$

Inequality (15) guarantees the uniqueness of the solution and its continuous dependance on the initial condition and forcing.

The boundary conditions are also physically appropriate, since the integration of transport equation (1) over domain $\mathbf{D}$ leads to a mass balance equation

$$
\begin{gathered}
\frac{\partial}{\partial t} \int_{\mathbf{D}} \phi d \mathbf{r}=\sum_{i=1}^{M} \int_{\mathbf{D}} f_{i}(\mathbf{r}, t) d \mathbf{r} \\
-\int_{S^{+} \cup S_{H}^{+}} U_{n} \phi d S-\int_{\mathbf{D}} \sigma \phi d \mathbf{r}-\int_{S_{0}} \nu^{s} \phi\left|\mathbf{e}_{3} \cdot \mathbf{n}\right| d S .
\end{gathered}
$$

Thus, the total mass of the pollutant increases due to the nonzero emission rates $f_{i}(\mathbf{r}, t)$, and decreases because of advective outflow of the pollutant through the boundary $S^{+} \cup S_{H}^{+}$and its chemical transformation $(\sigma \neq 0)$ and sedimentation on the ground $\left(\nu^{s} \neq 0\right)$.

For the numerical solution of the dispersion model (1)-(9), a balanced and absolutely stable second-order difference scheme, the splitting method, and the Crank-Nicholson schemes are used, [7].

\section{Positive systems of linear equations}

A system of linear equations $\Lambda \mathbf{x}=\mathbf{b}$ is called positive when the matrix $\Lambda$ is positive definite. By definition, $\Lambda$ is a positive definite $N \times N$ matrix if it is symmetric $\left(\Lambda=\Lambda^{t}\right)$ and $\mathbf{x}^{t} \Lambda \mathbf{x}>0$ for all $\mathbf{x} \in \mathbf{R}^{N}, \mathbf{x} \neq \mathbf{0}$. Let us consider the eigenvalue problem $\Lambda \mathbf{v}_{i}=\lambda_{i} \mathbf{v}_{i}$ for a positive definite matrix $\Lambda$. Hereafter, the eigenvalues of a real symmetric matrix $\Lambda$ are denoted as $\lambda_{1}, \lambda_{2}, \ldots, \lambda_{N}$, while $\mathbf{v}_{1}, \mathbf{v}_{2}, \ldots, \mathbf{v}_{N}$ are the corresponding orthonormal eigenvectors. 
Let us recall the main properties of solutions of positive systems of linear equations, which will be used in Sections 4 and 5 .

Property 1. If $\Lambda$ is a real symmetric matrix, then all of the eigenvalues of $\Lambda$ are real numbers, and the corresponding eigenvectors can be chosen so as to form an orthonormal basis in the Euclidean space $\mathbf{R}^{N}$.

Indeed, by the spectral theorem [5], $\Lambda$ can be factorized as $\Lambda=V \lambda V^{-1}$ where $V^{-1}=V^{t}$ is an orthogonal matrix with its column vectors as the orthonormal eigenvectors of $\Lambda$ and $\lambda=\operatorname{diag}\left\{\lambda_{1}, \lambda_{2}, \cdots, \lambda_{N}\right\}$ is the diagonal matrix formed by the eigenvalues of $\Lambda$.

Property 2. The eigenvalues of a positive definite matrix $\Lambda$ are positive numbers.

Indeed, let $\Lambda \mathbf{v}_{i}=\lambda_{i} \mathbf{v}_{i}$ where $\left\|\mathbf{v}_{i}\right\|=1$. Then $\mathbf{v}_{i}^{t} \Lambda \mathbf{v}_{i}=\lambda_{i} \mathbf{v}_{i}^{t} \mathbf{v}_{i}=\lambda_{i}\left\|\mathbf{v}_{i}\right\|^{2}$ and $\lambda_{i}=\mathbf{v}_{i}^{t} \Lambda \mathbf{v}_{i}>0$.

Property 3. Every positive definite matrix $\Lambda$ is a nonsingular matrix.

Indeed, it is well known that $\operatorname{det}(\Lambda)=\lambda_{1} \lambda_{2} \cdots \lambda_{N}$. Therefore, due to Property $2, \operatorname{det}(\Lambda)>0$.

Lemma 1. The unique solution of a positive system of linear equations $\Lambda \mathbf{x}=\mathbf{b}$ can be expressed as follows:

$$
\mathbf{x}=\sum_{i=1}^{N} \frac{\mathbf{v}_{i}^{t} \mathbf{b}}{\lambda_{i}} \mathbf{v}_{i}
$$

Proof. The linear system $\Lambda \mathbf{x}=\mathbf{b}$ has a unique solution because $\Lambda$ is a nonsingular matrix (see property 3 ), and hence, $\mathbf{x}=\Lambda^{-1} \mathbf{b}$ is such a solution. Using the factorization $\Lambda=V \lambda V^{-1}$ again, we obtain

$$
\mathbf{x}=\Lambda^{-1} \mathbf{b}=\left(V \lambda V^{-1}\right)^{-1} \mathbf{b}=\left(V \lambda^{-1}\right)\left(V^{t} \mathbf{b}\right) .
$$

The matrix $V \lambda^{-1}$ can be written by columns as follows:

$$
V \lambda^{-1}=\left(\frac{1}{\lambda_{1}} \mathbf{v}_{1}, \frac{1}{\lambda_{2}} \mathbf{v}_{2}, \ldots, \frac{1}{\lambda_{N}} \mathbf{v}_{N}\right),
$$


and the column vector $V^{t} \mathbf{b}$ is expressed in the form

$$
V^{t} \mathbf{b}=\left(\begin{array}{c}
\mathbf{v}_{1}^{t} \mathbf{b} \\
\mathbf{v}_{2}^{t} \mathbf{b} \\
\vdots \\
\mathbf{v}_{N}^{t} \mathbf{b}
\end{array}\right)
$$

Consequently, $\mathbf{x}=\left(V \lambda^{-1}\right)\left(V^{t} \mathbf{b}\right)=\sum_{i=1}^{N} \frac{\mathbf{v}_{i}^{t} \mathbf{b}}{\lambda_{i}} \mathbf{v}_{i}$.

The equation (17) allows estimating the sensitivity of the solution $\mathbf{x}$ of a linear system $\Lambda \mathbf{x}=\mathbf{b}$ with respect to small perturbations $\delta \mathbf{b}$ in the RHS $\mathbf{b}$.

Theorem 1. Let $\mathbf{x}$ be the solution of a positive system $\Lambda \mathbf{x}=\mathbf{b}$, and let $\delta \mathbf{b}$ be a variation in its $R H S \mathbf{b}$. If $\delta \mathbf{x}$ is the corresponding variation in the solution $\mathbf{x}$, then

$$
\|\delta \mathbf{x}\|^{2}=\sum_{i=1}^{N}\left(\frac{\mathbf{v}_{i}^{t} \delta \mathbf{b}}{\lambda_{i}}\right)^{2}
$$

where $\|\delta \mathbf{x}\|^{2}=(\delta \mathbf{x})^{t} \delta \mathbf{x}$.

Proof. The linear system $\Lambda(\mathbf{x}+\delta \mathbf{x})=\mathbf{b}+\delta \mathbf{b}$ is positive, and by Lemma 1 , $\mathbf{x}+\delta \mathbf{x}$ is written as

$$
\mathbf{x}+\delta \mathbf{x}=\sum_{i=1}^{N} \frac{\mathbf{v}_{i}^{t}(\mathbf{b}+\delta \mathbf{b})}{\lambda_{i}} \mathbf{v}_{i}=\sum_{i=1}^{N} \frac{\mathbf{v}_{i}^{t} \mathbf{b}}{\lambda_{i}} \mathbf{v}_{i}+\sum_{i=1}^{N} \frac{\mathbf{v}_{i}^{t} \delta \mathbf{b}}{\lambda_{i}} \mathbf{v}_{i}=\mathbf{x}+\sum_{i=1}^{N} \frac{\mathbf{v}_{i}^{t} \delta \mathbf{b}}{\lambda_{i}} \mathbf{v}_{i},
$$

and hence, $\delta \mathbf{x}=\sum_{i=1}^{N} \frac{\mathbf{v}_{i}^{t} \delta \mathbf{b}}{\lambda_{i}} \mathbf{v}_{i}$.

Since the eigenvectors are orthonormal, $\|\delta \mathbf{x}\|^{2}=(\delta \mathbf{x})^{t} \delta \mathbf{x}=\sum_{i=1}^{N}\left(\frac{\mathbf{v}^{t} \delta \mathbf{b}}{\lambda_{i}}\right)^{2}$.

\section{Corollary 1.}

$$
\|\delta \mathbf{x}\| \leq \frac{\sqrt{N}}{\lambda_{1}}\|\delta \mathbf{b}\|
$$

Proof. Let $0<\lambda_{1} \leq \lambda_{2} \leq \ldots \leq \lambda_{N}$. Using the Schwarz inequality $\left|\mathbf{v}_{i}^{t} \delta \mathbf{b}\right| \leq\left\|\mathbf{v}_{i}\right\|\|\delta \mathbf{b}\|$, we obtain

$$
\left(\frac{\mathbf{v}_{i}^{t} \delta \mathbf{b}}{\lambda_{i}}\right)^{2} \leq\left(\frac{\left\|\mathbf{v}_{i}\right\|\|\delta \mathbf{b}\|}{\lambda_{1}}\right)^{2} \leq\left(\frac{\|\delta \mathbf{b}\|}{\lambda_{1}}\right)^{2}
$$


for every index $i$. Since $\left\|\mathbf{v}_{i}\right\|^{2}=1$, (18) leads to

$$
\|\delta \mathbf{x}\|^{2}=\sum_{i=1}^{N}\left(\frac{\mathbf{v}_{i}^{t} \delta \mathbf{b}}{\lambda_{i}}\right)^{2} \leq \sum_{i=1}^{N}\left(\frac{\|\delta \mathbf{b}\|}{\lambda_{1}}\right)^{2}=\frac{\|\delta \mathbf{b}\|^{2}}{\lambda_{1}^{2}} N .
$$

The last estimate implies (19).

The inequality (19) indicates that the linear system is well-conditioned ([12]) if the ratio $\sqrt{N} / \lambda_{1}$ is not large. This estimate will be used in Section 5 to assess the quality of the calculated intensity parameters.

\section{Method for estimating the intensity of an unknown source}

Now, suppose a point source emits a pollutant into the atmosphere as $N$ successive instantaneous emissions:

$$
Q(t)=\sum_{i=1}^{N} Q_{i} \delta\left(t-t_{i}\right),
$$

where the Dirac function $\delta$ sets a unit impulse at each moment $t_{i}$. It is also assumed that the site of emission $\mathbf{r}_{0}$ and the moments $t_{i}$ are known. The goal is to develop a method for estimating the emission rates $Q_{i}$ using the time series of the concentration of the pollutant $\left\{\phi_{d}\left(\mathbf{R}, \tau_{k}\right)\right\}$, obtained at the monitoring site $\mathbf{R} \in \mathbf{D}$ and at times $\tau_{k} \in(0, T)$. To describe the method, we write the dispersion model (1) - (9) in the operator form

$$
\frac{\partial \phi}{\partial t}+A \phi=f(\mathbf{r}, t)+Q(t) \delta\left(\mathbf{r}-\mathbf{r}_{0}\right), \quad \phi(\mathbf{r}, 0)=\phi^{0}(\mathbf{r}) \text { in } \mathbf{D},
$$

where $A$ is defined by (13) and boundary conditions, $\phi(\mathbf{r}, t)$ is the concentration of the pollutant at the point $\mathbf{r} \in \mathbf{D}$ and at the moment $t \in(0, T), f(\mathbf{r}, t)$ describes the emission rates of known sources located in the domain $\mathbf{D}, \phi^{0}(\mathbf{r})$ is the distribution of the pollutant at the initial moment $t=0, Q(t) \delta\left(\mathbf{r}-\mathbf{r}_{0}\right)$ represents the unknown point source, $Q(t)$ is given by (20), and $\delta\left(\mathbf{r}-\mathbf{r}_{0}\right)$ is the Dirac delta function centered at the emission point $\mathbf{r}_{0} \in \mathbf{D}$.

Since the dispersion model (21) is linear, its solution $\phi(\mathbf{r}, t)$ can be represented as

$$
\phi(\mathbf{r}, t)=C_{0}(\mathbf{r}, t)+C(\mathbf{r}, t)+\sum_{i=1}^{N} Q_{i} C_{i}(\mathbf{r}, t),
$$


where $C_{0}$ is the solution of the problem

$$
\frac{\partial C_{0}}{\partial t}+A C_{0}=0, \quad C_{0}(\mathbf{r}, 0)=\phi^{0}(\mathbf{r}) \text { in } \mathbf{D}
$$

$C$ is the solution of the problem

$$
\frac{\partial C}{\partial t}+A C=f(\mathbf{r}, t), \quad C(\mathbf{r}, 0)=0 \text { in } \mathbf{D},
$$

and for each $i(i=1, \ldots, N), C_{i}$ is the solution of the problem

$$
\frac{\partial C_{i}}{\partial t}+A C_{i}=\delta\left(t-t_{i}\right) \delta\left(\mathbf{r}-\mathbf{r}_{0}\right), \quad C_{i}(\mathbf{r}, 0)=0 \text { in } \mathbf{D} .
$$

Note that all solutions to problems (23)-(25) can be calculated in advance, regardless of the intensity parameters $Q_{i}$.

The method for estimating the emission rates $Q_{i}$ is formulated as an unconstrained minimization problem:

$$
\begin{gathered}
\operatorname{Min} \Phi=\frac{1}{2} \int_{0}^{T}\left[\phi_{d}(\mathbf{R}, t)-C_{0}(\mathbf{R}, t)-C(\mathbf{R}, t)-\sum_{j=1}^{N} Q_{j} C_{j}(\mathbf{R}, t)\right]^{2} d t \\
+\frac{\varepsilon}{2} \sum_{j=1}^{N} Q_{j}^{2},
\end{gathered}
$$

where $\varepsilon$ is a positive parameter.

Without loss of generality, it is assumed that the concentration of the pollutant $\phi_{d}$ at monitoring site $\mathbf{R}$ is known as a function of the continuous time variable $t \in(0, T)$. The minimization process allows the function $\phi(\mathbf{R}, t)$ to approach the concentration data $\phi_{d}(\mathbf{R}, t)$ by decreasing the first (integral) term of the function $\Phi$, while the sum of the squares of the parameters $Q_{i}$ (the second term of the function $\Phi$ ) is kept as small as possible. Note that $\varepsilon$ is a weighting factor that affects the minimization process. For small values of $\varepsilon$, the minimization process focuses on decreasing the integral term of the function $\Phi$, i.e., on approximating $\phi(\mathbf{R}, t)$ to the concentration data $\phi_{d}(\mathbf{R}, t)$. On the other hand, for large values of $\varepsilon$, the minimization process focuses on decreasing the second term of the function $\Phi$, and therefore the values $Q_{i}$ of the solution to problem (26) are close to zero. The following simple example explains this behavior. Let $\Phi(x)=\frac{1}{2}(d-x)^{2}+\frac{\varepsilon}{2} x^{2}$ be a real function. The minimum of $\Phi$ is attained at the point $x_{\varepsilon}=\frac{d}{1+\varepsilon}$. Thus, $x_{\varepsilon}$ tends to $d$ as $\varepsilon$ tends to zero, that 
is, the first term of the function $\Phi$ is minimized. However, $x_{\varepsilon} \rightarrow 0$ as $\varepsilon \rightarrow \infty$, i.e., the second term of the function $\Phi$ is minimized. The $\varepsilon$ parameter has some additional advantages which will be presented below.

The necessary condition for minimizing the function $(26)(\nabla \Phi=\mathbf{0})$ requires solving the system of equations:

$$
\begin{gathered}
\frac{\partial \Phi}{\partial Q_{i}}=\sum_{j=1}^{N} Q_{j} \int_{0}^{T} C_{i}(\mathbf{R}, t) C_{j}(\mathbf{R}, t) d t+\varepsilon Q_{i}- \\
\int_{0}^{T}\left[\phi_{d}(\mathbf{R}, t)-C_{0}(\mathbf{R}, t)-C(\mathbf{R}, t)\right] C_{i}(\mathbf{R}, t) d t=0,
\end{gathered}
$$

for $i=1, \ldots, N$.

In matrix form, this system of linear equations can be written as

$$
\Lambda \mathbf{Q}=\mathbf{b},
$$

where $\Lambda=G+\varepsilon I, G$ is the square real matrix with entries

$$
G_{i j}=\int_{0}^{T} C_{i}(\mathbf{R}, t) C_{j}(\mathbf{R}, t) d t \quad(i, j=1, . ., N),
$$

$I$ is the identity matrix of order $N, \mathbf{Q}=\left(Q_{1}, \ldots, Q_{N}\right)^{t}$ is the column vector that contains the unknown intensity parameters, and $\mathbf{b}$ is the RHS column-vector with components $\mathbf{b}_{i}=\int_{0}^{T}\left[\phi_{d}(\mathbf{R}, t)-C_{0}(\mathbf{R}, t)-C(\mathbf{R}, t)\right] C_{i}(\mathbf{R}, t) d t, i=1, \ldots, N$. In the particular case when the unknown point source is the only pollution source in $\mathbf{D}$ then $C_{0}(\mathbf{r}, t)=C(\mathbf{r}, t)=0$, since $f=0$ and $\phi^{0}=0$, and each component of vector $\mathbf{b}$ of system (27) is reduced to $\mathbf{b}_{i}=\int_{0}^{T} \phi_{d}(\mathbf{R}, t) C_{i}(\mathbf{R}, t) d t$, $i=1, \ldots, N$.

Since $G$ is a real symmetric $N \times N$ matrix, $\Lambda$ is also a real symmetric $N \times N$ matrix. The following theorems establish other important features of matrix $\Lambda$ and linear system $\Lambda \mathbf{Q}=\mathbf{b}$.

Theorem 2. For every $\varepsilon>0$, the matrix $\Lambda=G+\varepsilon I$ is positive definite. 
Proof. The symmetric matrix $G$ is positive semidefinite since

$$
\begin{aligned}
& \mathbf{x}^{t} G \mathbf{x}=\sum_{i=1}^{N} \sum_{j=1}^{N} x_{i} x_{j} \int_{0}^{T} C_{i}(\mathbf{R}, t) C_{j}(\mathbf{R}, t) d t \\
= & \int_{0}^{T}\left[x_{1} C_{1}(\mathbf{R}, t)+\cdots+x_{N} C_{N}(\mathbf{R}, t)\right]^{2} d t \geq 0,
\end{aligned}
$$

for $\mathbf{x} \in \mathbf{R}^{N}$.

Therefore, for every $\varepsilon>0, \mathbf{x}^{t} \Lambda \mathbf{x}=\mathbf{x}^{t} G \mathbf{x}+\varepsilon \mathbf{x}^{t} \mathbf{x} \geq \varepsilon\|\mathbf{x}\|^{2}>0$ for all $\mathbf{x} \in \mathbf{R}^{N}$, $\mathbf{x} \neq \mathbf{0}$.

Theorem 3. The solution of linear system (27) is the global minimum of function $\Phi$.

Proof. Due to Theorem 2, the matrix $\Lambda$ is nonsingular (see property 3 ). Thus, for every $\varepsilon>0$, the system (27) has a unique solution $\mathbf{Q}_{\varepsilon}$. Moreover, $\Phi$ is a strictly convex function because its Hessian $\mathbf{H}=\left(\partial^{2} \Phi / \partial Q_{i} \partial Q_{j}\right)_{N \times N}=\Lambda$ is a positive definite matrix [6]. Consequently, $\mathbf{Q}_{\varepsilon}$ minimizes the function $\Phi$, since $\nabla \Phi\left(\mathbf{Q}_{\varepsilon}\right)=\mathbf{0}$ and $\mathbf{H}\left(\mathbf{Q}_{\varepsilon}\right)=\Lambda$ is a positive definite matrix, [6]. Furthermore, since $\Phi$ is a strictly convex function, then $\mathbf{Q}_{\varepsilon}$ is the unique global minimum of function $\Phi,[6]$.

Since the matrix $G$ can be singular, the introduction of parameter $\varepsilon$ in the optimization problem (26) is necessary to assure a solution of linear system (27). Such solution denoted as $\mathbf{Q}_{\varepsilon}$, is the solution of the parameter estimation problem.

We now discuss the sensitivity of such a solution, as well as the criterion for choosing the parameter $\varepsilon$. According to property 1 , the eigenvalues $\lambda_{1}, \lambda_{2}, \ldots, \lambda_{N}$ of matrix $G$ are real numbers and the corresponding eigenvectors $\mathbf{v}_{1}, \mathbf{v}_{2}, \ldots, \mathbf{v}_{N}$ form an orthonormal basis for space $\mathbf{R}^{N}$. Moreover, $G$ is a positive semidefinite matrix and therefore $\lambda_{i} \geq 0$ for all $i$ (see the proofs of Property 2 and Theorem 2). Further, the eigenvalues of matrix $\Lambda$ are $\lambda_{1}+\varepsilon, \lambda_{2}+\varepsilon, \ldots, \lambda_{N}+\varepsilon$ and $\mathbf{v}_{1}, \mathbf{v}_{2}, \ldots, \mathbf{v}_{N}$ are the corresponding eigenvectors. Let us suppose that $0<\varepsilon \leq \lambda_{1}+\varepsilon \leq \lambda_{2}+\varepsilon \leq \cdots \leq \lambda_{N}+\varepsilon$. Due to Lemma 1 and inequality (19), the solution $\mathbf{Q}_{\varepsilon}$ is

$$
\mathbf{Q}_{\varepsilon}=\sum_{i=1}^{N} \frac{\mathbf{v}_{i}^{t} \mathbf{b}}{\lambda_{i}+\varepsilon} \mathbf{v}_{i}
$$


and

$$
\left\|\delta \mathbf{Q}_{\varepsilon}\right\| \leq \frac{\sqrt{N}}{\lambda_{1}+\varepsilon}\|\delta \mathbf{b}\|,
$$

where $\delta \mathbf{b}$ represents a variation in the RHS $\mathbf{b}$ of system (27), while $\delta \mathbf{Q}_{\varepsilon}$ is the corresponding variation in the solution $\mathbf{Q}_{\varepsilon}$. Note that $\delta \mathbf{b}$ is usually present in system (27) due to errors in the data on the concentration of pollutant $\phi_{d}$. Also note that in inequality (29), the factor $\sqrt{N} /\left(\lambda_{1}+\varepsilon\right)$ is used to evaluate the quality of the intensity parameter computation. Indeed, if this coefficient is not a large number, then the error $\left\|\delta \mathbf{Q}_{\varepsilon}\right\|$ is controlled by the error $\|\delta \mathbf{b}\|$, that is, the quality of the solution $\mathbf{Q}_{\varepsilon}$ is comparable to the quality of the data used in $\mathbf{b}$. But if $\lambda_{1}$ is a very small number, one can use a positive parameter $\varepsilon$ in order to avoid division by this number and, therefore, prevent the growth of errors in the solution. For example, if $N=10, \lambda_{1}=10^{-6}$ and $\varepsilon=0$, then $\sqrt{N} /\left(\lambda_{1}+\varepsilon\right)=10^{6} \sqrt{10}$, and therefore, according to (29), the error $\left\|\delta \mathbf{Q}_{\varepsilon}\right\|$ can be huge. However, if the value of parameter $\varepsilon$ is taken as $\varepsilon=0.1$, then $\sqrt{N} /\left(\lambda_{1}+\varepsilon\right) \approx 10 \sqrt{10}$, and the error $\left\|\delta \mathbf{Q}_{\varepsilon}\right\|$ is under control. Conversely, if $\lambda_{1}$ is not too small, then the parameter $\varepsilon$ can be taken as a very small number or equal to zero.

\section{Parameter estimation example}

To demonstrate the capabilities of the new method, we now consider a simple example of parameter estimation. Let us assume that the concentration of the pollutant is calculated using the one-dimensional dimensionless version of the dispersion model (1) - (9):

$$
\begin{gathered}
\frac{\partial \phi}{\partial t}+u \frac{\partial \phi}{\partial x}-\mu \frac{\partial^{2} \phi}{\partial x^{2}}+\sigma \phi=Q(t) \delta\left(x-x_{0}\right), 0<x<L, 0<t<T, \\
\mu \frac{\partial \phi}{\partial x}(0, t)-u \phi(0, t)=0,0<t, \\
\mu \frac{\partial \phi}{\partial x}(L, t)=0,0<t, \\
\phi(x, 0)=0,0<x<L .
\end{gathered}
$$

Here, $\phi(x, t)$ is the concentration of the pollutant in the domain $\mathbf{D}=(0, L)$ and time interval $(0, T)$. The positive parameters $u, \mu$ and $\sigma$ are the wind velocity, the diffusion coefficient and the pollutant transformation rate, respectively. 
Point $x_{0}$ is the emission site, and instantaneous emissions occur at times $t_{i}$, i.e., emission rate $Q(t)$ is determined by formula (20):

$$
Q(t)=\sum_{i=1}^{N} Q_{i} \delta\left(t-t_{i}\right) .
$$

It is also assumed that the point source located at $x_{0}$ is the only source of pollution in $\mathbf{D}$, and therefore $\phi^{0}=0$. We take $u=0.25, \mu=0.05, \sigma=0.001$, $L=1, T=40, x_{0}=0.2$, and $R=0.8$ is the monitoring site. It is assumed that there were five instantaneous emissions in $(0, T)(N=5)$, which occurred at the moments $t_{1}=1, t_{2}=5, t_{3}=7, t_{4}=12$ and $t_{5}=14$.

Since $\phi^{0}=0$ and $f=0$, the solutions of problems (23) and (24) are equal to zero: $C_{0}=0$ and $C=0$. For the monitoring point $x=R$, solutions $C_{i}(x, t)$ to problems $(25), i=1, \ldots, 5$, are shown in Figure 1. It can be seen that the graphics $C_{i}(R, t), i=2, \ldots, 5$, are the translation of the curve $C_{1}(R, t)$. This is due to the use of constant coefficients in the transport equation (30), which simulate the steady-state conditions of dispersion in the atmosphere.

To test the efficiency of the parameter estimation method, the following intensity parameters $Q_{i}$ were set: $Q_{1}=10, Q_{2}=25, Q_{3}=3, Q_{4}=15$ and $Q_{5}=$ 20. According to equation (22), these parameters determine the concentration of the pollutant at the monitoring point $x=R: \phi(R, t)=\sum_{i=1}^{5} Q_{i} C_{i}(R, t)$. Thus, the synthetic concentration data can be written as $\phi_{d}(R, t)=\phi(R, t)+\delta \phi(R, t)$, where $\delta \phi(R, t)$ is a small variation. In this example, $\delta \phi(R, t)=s * \max \phi(R, t) *$ rand $[-0.5,0.5]$, where "rand" is the function that generates random values uniformly distributed in the interval $[-0.5,0.5]$, and the coefficient $s$ determines the amplitude of variations.

Figures 2, 3 and 4 show the synthetical concentration data obtained for $s=0, s=0.05$ and $s=0.1$, respectively. The intensity parameters are now estimated for each of these three datasets.

The components of the vector $\mathbf{b}$ and the entries $G_{i j}$ of the matrix $G$ of the linear system (27) are calculated using the formulas

$$
\mathbf{b}_{i}=\int_{0}^{T} \phi_{d}(R, t) C_{i}(R, t) d t, i=1, \ldots, 5,
$$

and

$$
G_{i j}=\int_{0}^{T} C_{i}(R, t) C_{j}(R, t) d t, i, j=1, . ., 5 .
$$




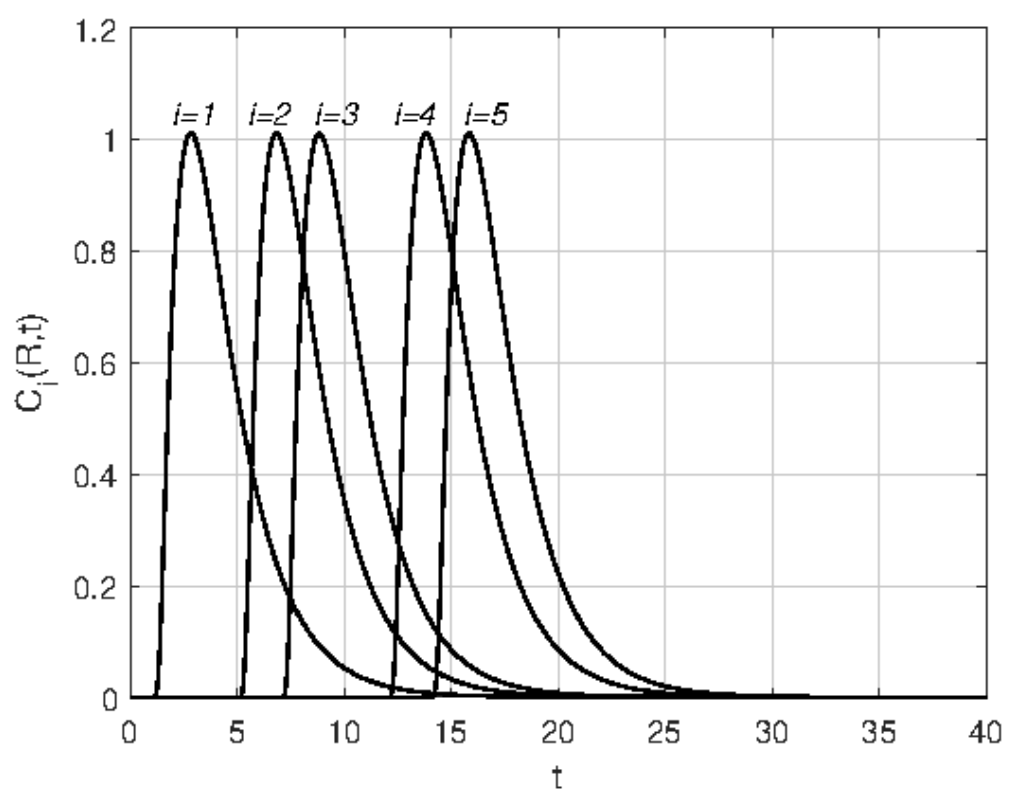

Figure 1: The solutions $C_{i}(x, t)$ of problems (25) at the monitoring point $x=R$.

In addition, the matrix $\lambda=\operatorname{diag}\left\{\lambda_{1}, \lambda_{2}, \cdots, \lambda_{N}\right\}$ formed from the eigenvalues of matrix $G$, and the matrix $V$ formed from the corresponding eigenvectors $\mathbf{v}_{i}$ of $G$ (see (28)), were obtained using the MATLAB function eig, [8]:

$$
\begin{aligned}
\lambda & =\left(\begin{array}{ccccc}
0.95 & 0 & 0 & 0 & 0 \\
0 & 1.10 & 0 & 0 & 0 \\
0 & 0 & 2.47 & 0 & 0 \\
0 & 0 & 0 & 3.99 & 0 \\
0 & 0 & 0 & 0 & 4.76
\end{array}\right) \text { and } \\
V & =\left(\begin{array}{ccccc}
0.13 & -0.12 & 0.91 & -0.26 & 0.23 \\
-0.56 & 0.44 & -0.11 & -0.43 & 0.53 \\
0.57 & -0.34 & -0.37 & -0.32 & 0.54 \\
-0.44 & -0.55 & 0.02 & 0.54 & 0.44 \\
0.37 & 0.59 & 0.09 & 0.58 & 0.39
\end{array}\right)
\end{aligned}
$$

Since the smallest eigenvalue is not a small number $\left(\lambda_{1}=0.9523\right)$, the 


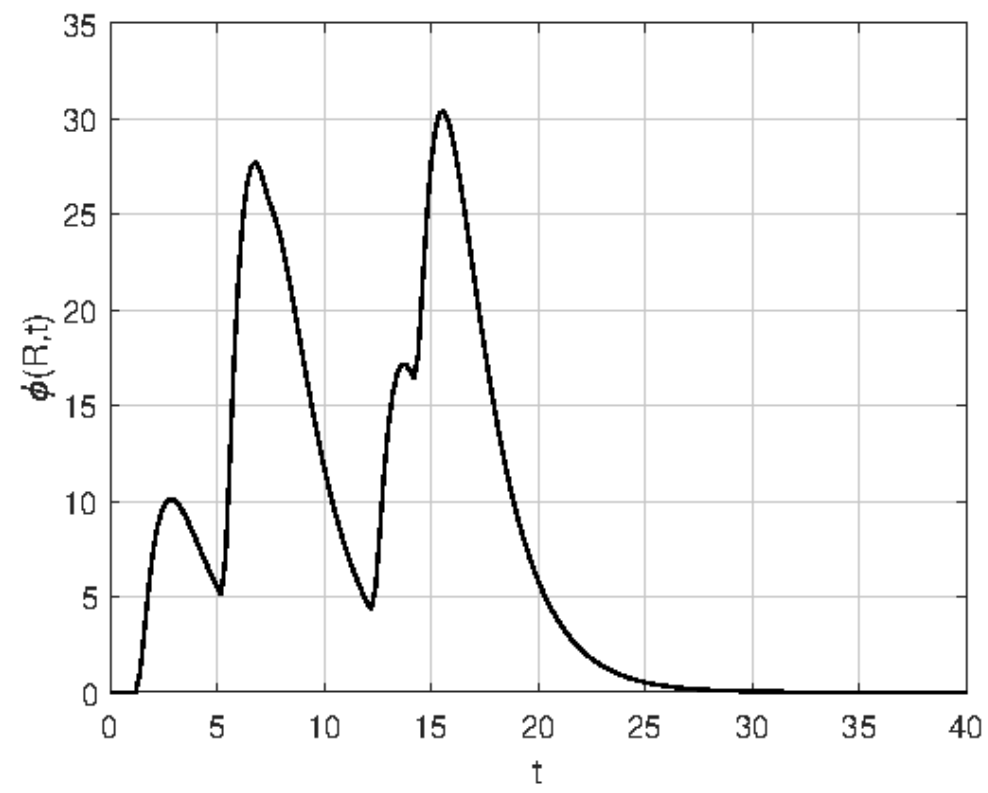

Figure 2: The synthetical concentration data $\phi_{d}(R, t)=\phi(R, t)$ for the parameter $s=0$.

parameter $\varepsilon$ is chosen as a small value: $\varepsilon=10^{-6}$. Thus, the ratio $\sqrt{N} /\left(\lambda_{1}+\varepsilon\right)=$ 2.34 , i.e., problem (27) is well-conditioned, and by (29), the error $\left\|\delta \mathbf{Q}_{\varepsilon}\right\|$ is of the same order as the error $\|\delta \mathbf{b}\|$. That is, the solution $\mathbf{Q}_{\varepsilon}$ is of the same quality as the data. To verify this, the three experiments are considered with different values of $s: s=0, s=0.05$ and $s=0.1$. For $s=0, \phi_{d}(R, t)=\phi(R, t)$ (see Figure 2), that is, the relative error in $\phi$ is zero $\left(\left\|\phi_{d}-\phi\right\| /\|\phi\|=0\right)$ and $\delta \mathbf{b}=\mathbf{0}$. The solution of linear system $(27)$ is $\mathbf{Q}_{\varepsilon}=\mathbf{Q}=(10,25,3,15,20)^{t}$, and its relative error is also zero $\left(\left\|\mathbf{Q}_{\varepsilon}-\mathbf{Q}\right\| /\|\mathbf{Q}\|=0\right)$, as it should be. For $s=0.05$, the concentration data $\phi_{d}$ are shown in Figure 3 . In this case, the relative error in data is $\left\|\phi_{d}-\phi\right\| /\|\phi\|=0.0378$ (3.7\%), while the solution $\mathbf{Q}_{\varepsilon}=$ $(9.99,24.99,2.98,15.02,20.01)^{t}$ of the linear system $(27)$ has the relative error $\left\|\mathbf{Q}_{\varepsilon}-\mathbf{Q}\right\| /\|\mathbf{Q}\|=0.0008(0.08 \%)$. Note that this error decreases because $\lambda_{i}>$ 1 , for $i=2, \ldots, 5$ (see equation (18)). Finally, Figure 4 shows the concentration data $\phi_{d}$ for $s=0.1$. In this case, the relative error in data is $\left\|\phi_{d}-\phi\right\| /\|\phi\|=$ 0.0756 (7.5\%), while the solution $\mathbf{Q}_{\varepsilon}=(9.98,24.99,2.97,15.04,20.02)^{t}$ of the linear system (27) has the relative error $\left\|\mathbf{Q}_{\varepsilon}-\mathbf{Q}\right\| /\|\mathbf{Q}\|=0.0017$ (0.17\%). As 


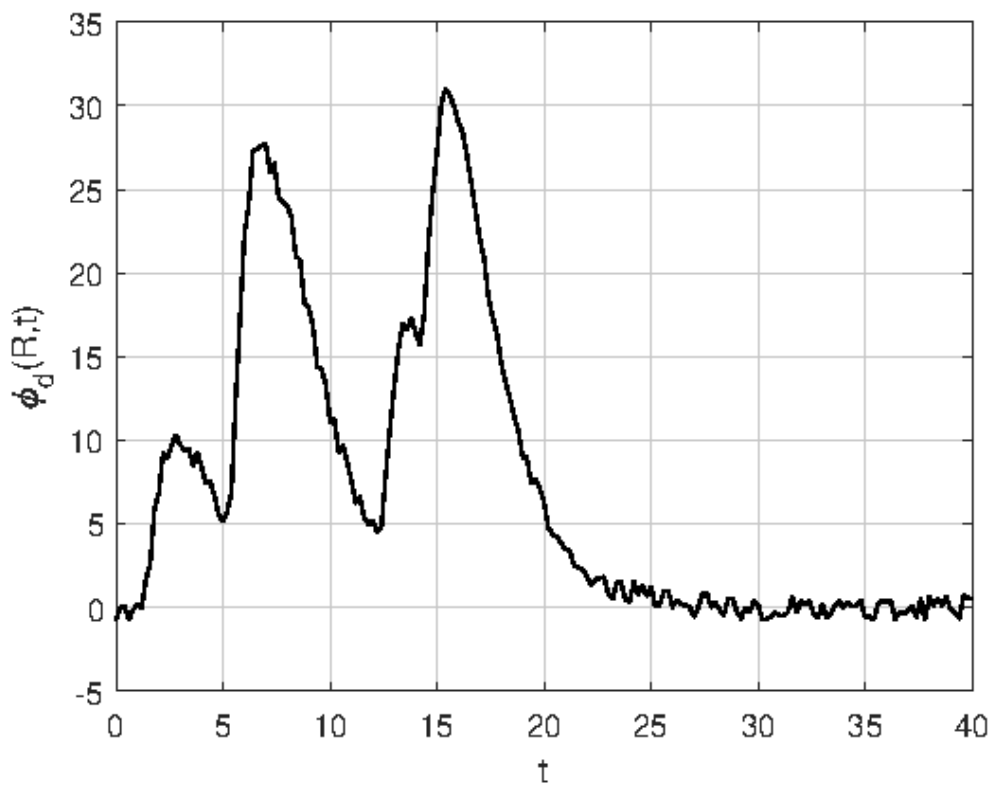

Figure 3: The synthetical concentration data $\phi_{d}(R, t)$ for the parameter $s=0.05$.

in the previous case, the relative error decreases because the eigenvalues $\lambda_{i}$, $i=2, \ldots, 5$, are greater than 1 .

As one can see, this method for estimating the emission rates is efficient and economical, since the problem of recovering the intensity parameters $Q_{i}$ is reduced to the formulation and solution of a positive system of linear equations of order $N$.

\section{Acknowledgments}

This research was supported by the grants 25170 and 14539 of the National System of Investigators (SNI-CONACyT, México). 


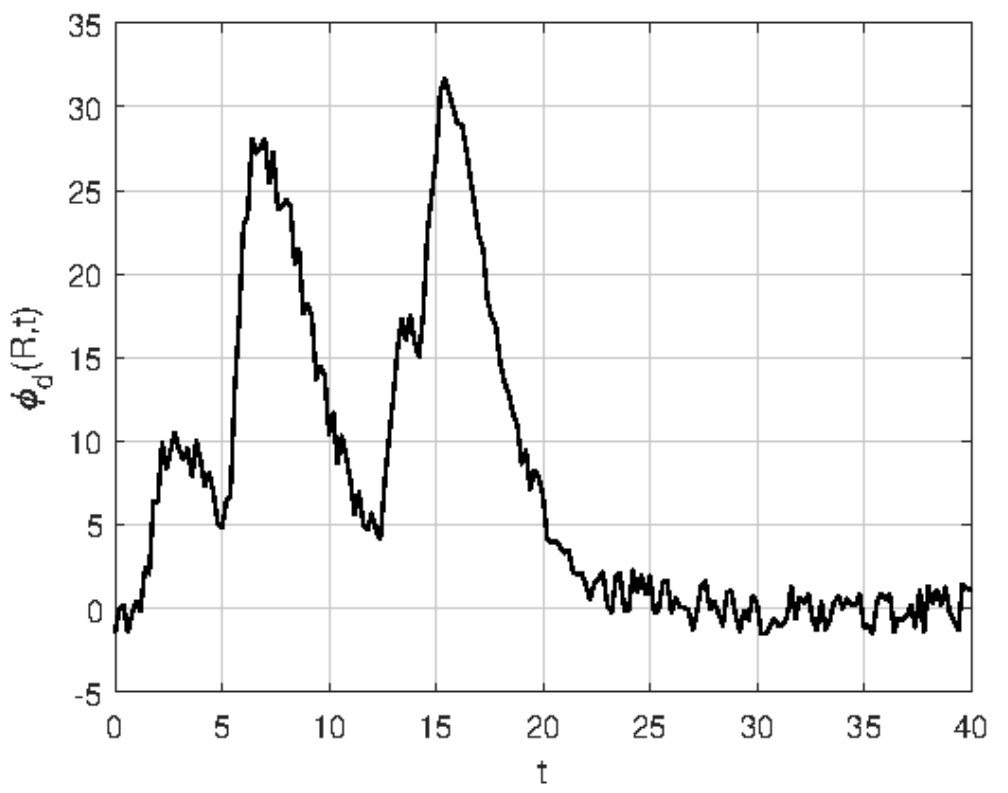

Figure 4: The synthetical concentration data $\phi_{d}(R, t)$ for the parameter $s=0.1$.

\section{References}

[1] T. Chai, R. Draxler and A. Stein, Source term estimation using air concentration measurements and a Lagrangian dispersion model - experiments with pseudo and real Cesium-137 observations from the Fukushima nuclear accident, Atmospheric Environment, 106 (2015), 241-251.

[2] I.G. Enting, Inverse Problems in Atmospheric Constituent Transport, Cambridge University Press, Cambridge (2002).

[3] C.A. Gough, M.J. Chadwick, B. Biewald, J. Kylenstierna, P.D. Bailey and S. Cinderby, Developing optimal abatement strategies for the effects of sulphur and nitrogen deposition at European scale, Water, Air and Soil Pollution, 85, No 4 (1995), 2601-2606.

[4] J. Hadamard, Lectures on Cauchy's Problem in Linear Partial Differential Equations, Dover Publications (1923). 
[5] S. Lang, Linear Algebra, Springer, New York (1987).

[6] D.G. Luenberger, Linear and Nonlinear Programming, Addison-Wesley, Mass. (1984).

[7] G.I. Marchuk, Mathematical Models in Environmental Problems, Elsevier, New York (1986).

[8] J.H. Mathews and K.D. Fink, Numerical Methods Using Matlab, Pearson, New Jersey (2004).

[9] H. Nagai, G. Katata, H. Terada and M. Chino, Source term estimation of ${ }^{131} \mathrm{I}$ and ${ }^{137} \mathrm{Cs}$ discharged from the Fukushima Daiichi Nuclear Power Plant into the atmosphere. In: S. Takahashi (Ed.), Radiation Monitoring and Dose Estimation of the Fukushima Nuclear Accident, Springer, Tokyo (2014), 155-173.

[10] D. Parra-Guevara, Y.N. Skiba and D. Peña-Maciel, Controlling the forcing of the linear transport equation to meet air quality norms at every point. International Journal of Applied Mathematics, 30, No 6 (2017), 527-545; DOI: 10.12732/ijam.v30i6.6.

[11] D. Parra-Guevara and Y.N. Skiba, Elements of the mathematical modelling in the control of pollutants emissions, Ecological Modelling, 167, No 3 (2003), 263-275.

[12] A. Ralston and P. Rabinowitz, A First Course in Numerical Analysis, Dover, New York (2001).

[13] J.H. Seinfeld and S.N. Pandis, Atmospheric Chemistry and Physics: From Air Pollution to Climate Change, Wiley, New York (1998).

[14] Y.N. Skiba and D. Parra-Guevara, Industrial pollution transport. Part I: Formulation of the problem and air pollution estimates. Env. Modeling and Assessment, 5, No 3 (2000), 169-175.

[15] K. Wark, W.T. Davis and C.F. Warner, Air Pollution: Its Origin and Control, Addison-Wesley, California (1999). 